\title{
A RIEMANN TYPE DEFINITION OF A VARIATIONAL INTEGRAL
}

\author{
WASHEK F. PFEFFER
}

(Communicated by Andrew M. Bruckner)

\begin{abstract}
We present a Riemann type definition of a coordinate free integral for which a general divergence theorem holds. The definition is particularly simple in dimension one.
\end{abstract}

In $\left[\mathrm{P}_{2}\right]$ we defined a coordinate free variational integral on bounded sets of finite perimeter, and used it to establish a very general Gauss-Green theorem. In this note we show (Corollary 3.4) that the variational integral of $\left[\mathrm{P}_{2}\right]$ has a simple Riemann type definition, appreciably more transparent than that of $\left[\mathrm{P}_{2}\right.$, §7]. In dimension one, it enlightens the relationship between the variational integral of $\left[\mathrm{P}_{2}\right]$ and the generalized Riemann integral of Henstock and Kurzweil (see $\left[\mathrm{P}_{2}\right.$, Definition 2.1]).

\section{Preliminaries}

All functions we consider are real-valued. If $f$ is a function on a set $A$ and $B \subset A$, we denote by $f\lceil B$ the restriction of $f$ to $B$; when no confusion can arise we write $f$ instead of $f \nmid B$.

Throughout this note, $m \geq 1$ is a fixed integer. The set of all real numbers is denoted by $\mathbf{R}$, and the $m$-fold Cartesian product of $\mathbf{R}$ is denoted by $\mathbf{R}^{m}$. For $x=\left(\xi_{1}, \ldots, \xi_{m}\right)$ and $\varepsilon>0$, we let $|x|=\max \left\{\left|\xi_{1}\right|, \ldots,\left|\xi_{m}\right|\right\}$ and $U(x, \varepsilon)=$ $\left\{y \in \mathbf{R}^{m}:|x-y|<\varepsilon\right\}$. If $E \subset \mathbf{R}^{m}$, then $\operatorname{cl} E$, int $E$, bd $E$, and $|E|$ denote, respectively, the closure, interior, boundary, and outer Lebesgue measure of $E$; furthermore $d(E)=\sup \{|x-y|: x, y \in E\}$.

Let $E \subset \mathbf{R}^{m}$. We say that an $x \in \mathbf{R}^{m}$ is, respectively, a density or dispersion point of $E$ whenever

$$
\liminf _{\varepsilon \rightarrow 0+} \frac{|E \cap U(x, \varepsilon)|}{(2 \varepsilon)^{m}}=1 \text { or } \limsup _{\varepsilon \rightarrow 0+} \frac{|E \cap U(x, \varepsilon)|}{(2 \varepsilon)^{m}}=0 \text {. }
$$

The set of all density points of $E$ is called the essential interior of $E$, denoted by $\operatorname{int}_{e} E$, and the set of all nondispersion points of $E$ is called the essential closure of $E$, denoted by $\mathrm{cl}_{e} E$. The essential boundary of $E$ is the set $\mathrm{bd}_{e} E=$ $\mathrm{cl}_{e} E-\operatorname{int}_{e} E$. Clearly int $E \subset \operatorname{int}_{e} E \subset \operatorname{cl}_{e} E \subset \operatorname{cl} E$, and so $\mathrm{bd}_{e} E \subset$ bd $E$. If

Received by the editors June 5, 1990 .

1980 Mathematics Subject Classification (1985 Revision). Primary 26A39, $26 \mathrm{~B} 15$. 
$\mathrm{cl}_{e} E$ equals $E$ or $\mathrm{cl} E$, the set $E$ is called essentially closed or nondispersed, respectively.

The $(m-1)$-dimensional outer Hausdorff measure $\mathscr{H}$ in $\mathbf{R}^{m}$ is defined so that it is the counting measure if $m=1$, and agrees with the Lebesgue measure in $\mathbf{R}^{m-1}$ if $m>1$. A bounded set $A \subset \mathbf{R}^{m}$ is called a $B V$ set $(B V$ for bounded variation) whenever the number $\|A\|=\mathscr{H}\left(\mathrm{bd}_{e} A\right)$, called the perimeter of $A$, is finite. By [Fe, $\S 2.10 .6$ and Theorem 4.5.11], the family $B V$ of all $B V$ sets coincides with the collection of all bounded measurable subsets of $\mathbf{R}^{m}$ whose De Giorgi's perimeter in $\mathbf{R}^{m}$, defined in [M-M, $\left.§ 2.1 .2\right]$, is finite. If $E \subset \mathbf{R}^{m}$ we denote by $B V_{E}$ the family of all $B V$ subsets of $E$.

We shall need a lemma proved by G. Congedo and I. Tamanini (cf. [C-T] and $[\mathrm{T}-\mathrm{G}]$, or $\left[\mathrm{P}_{2}\right.$, Proposition 3.2]).

Lemma 1.1. For each $B V$ set $A$ there are nondispersed sets $A_{n} \in B V_{A}$ such that $\left\|A_{n}\right\| \leq\|A\|$ and $\left|A-A_{n}\right| \leq\|A\| / n, n=1,2, \ldots$.

Let $A \in B V$. An $x \in \mathbf{R}^{m}$ is called a perimeter dispersion point of $A$ whenever

$$
\lim _{\varepsilon \rightarrow 0+} \frac{\mathscr{H}\left[\mathrm{bd}_{e} A \cap U(x, \varepsilon)\right]}{(2 \varepsilon)^{m-1}}=0 .
$$

The set of all $x \in \operatorname{int}_{e} A$ which are perimeter dispersion points of $A$ is called the critical interior of $A$, denoted by $\operatorname{int}_{c} A$. It has been proved in [V, $\left.\S 4\right]$, that $\mathscr{H}\left(\operatorname{int}_{e} A-\operatorname{int}_{c} A\right)=0$. The regularity of $A$ is the number

$$
r(A)= \begin{cases}\frac{|A|}{d(A)\|A\|} & \text { if } d(A)\|A\|>0, \\ 0 & \text { otherwise. }\end{cases}
$$

The usual concept of regularity defined in [S, Chapter $4, \S 2]$ is related to $r(A)$ by the isoperimetric inequality (see [M-M, §2.2]).

\section{PARTitions}

A dyadic cube is the product $\prod_{i=1}^{m}\left[k_{i} 2^{-n},\left(k_{i}+1\right) 2^{-n}\right)$ where $k_{1}, \ldots, k_{m}$ and $n$ are integers with $n \geq 0$. Given a function $\delta$ on a set $E$, we let $N_{\delta}=\{x \in E: \delta(x)=0\}$ and call it the null set of $\delta$. A caliber is any sequence $\eta=\left\{\eta_{j}\right\}$ of positive real numbers. A set $N \subset \mathbf{R}^{m}$ is called thin if its $\mathscr{H}$ measure is $\sigma$-finite.

Let $A \in B V$. A nonnegative function $\delta$ on $\operatorname{cl}_{e} A$ is called a gage in $A$ whenever its null set $N_{\delta}$ is thin. A partition in $A$ is a collection (possibly empty) $P=\left\{\left(A_{1}, x_{1}\right), \ldots,\left(A_{p}, x_{p}\right)\right\}$ where $A_{1}, \ldots, A_{p}$ are disjoint $B V$ subsets of $A$ and $x_{i} \in \operatorname{cl}_{e} A_{i}, i=1, \ldots, p$; the set $\bigcup_{i=1}^{p} A_{i}$ is called the body of $P$, denoted by $\bigcup P$.

Definition 2.1. Let $\varepsilon>0$, let $\eta$ be a caliber, and let $\delta$ be a gage in a $B V$ set $A$. We say that a partition $P=\left\{\left(A_{1}, x_{1}\right), \ldots,\left(A_{p}, x_{p}\right)\right\}$ in $A$ is:

(1) dyadic if $A_{1}, \ldots, A_{p}$ are dyadic cubes;

(2) $\varepsilon$-regular if $r\left(A_{i}\right)>\varepsilon, i=1, \ldots, p$;

(3) $\delta$-fine if $d\left(A_{i}\right)<\delta\left(x_{i}\right), i=1, \ldots, p$;

(4) $(\varepsilon, \eta)$-approximating if $A-\bigcup P$ is the union of disjoint $B V$ sets $B_{1}, \ldots, B_{k}$ such that $\left\|B_{j}\right\|<1 / \varepsilon$ and $\left|B_{j}\right|<\eta_{j}, j=1, \ldots, k$. 
The family of all $\varepsilon$-regular $\delta$-fine $(\varepsilon, \eta)$-approximating partitions in $A$ is denoted by $\Pi(A, \varepsilon ; \delta, \eta)$.

Remark 2.2. Let $\delta$ be a gage in a $B V$ set $A$, and let $\delta_{+}=\delta\left\lceil\left(\mathrm{cl}_{e} A-N_{\delta}\right)\right.$. If $\varepsilon>0$ and $P$ is an $\varepsilon$-regular $\delta$-fine partition in $A$, then in the terminology of [P $\mathrm{P}_{2}$, Definition 7.1], $P$ is a tight $\delta_{+}$-fine $\varepsilon$-partition in $A \bmod N_{\delta}$.

Lemma 2.3. Let $K=\prod_{i=1}^{m}\left[k_{i}, k_{i}+2^{h}\right)$ where $k_{1}, \ldots, k_{m}$ and $h$ are integers, let $\delta$ be a gage in $K$, and let $\eta$ be a caliber. There is a $\tau>0$, depending only on the dimension $m$, such that a $\delta$-fine $(\tau, \eta)$-approximating dyadic partition in $K$ exists.

Proof. We employ the idea of E. J. Howard (cf. [H]). According to [Fa, Theorem 1.6(a)], there is a sequence $\left\{N_{j}\right\}$ of sets such that $N_{\delta}=\bigcup_{j} N_{j}$ and $\mathscr{H}\left(N_{j}\right)<2, j=1,2, \ldots$. Fix an integer $j \geq 1$. It follows from [Fa, Theorem 5.1] that there is an $\alpha>0$, depending only on the dimension $m$, and a countable family $\mathscr{C}_{j}$ of dyadic cubes of diameters less than $\eta_{j} / \alpha$ such that $N_{j} \subset \operatorname{int}\left(\bigcup \mathscr{C}_{j}\right)$ and $\sum_{C \in \mathscr{E}_{j}}[d(C)]^{m-1}<\alpha$. Thus if $\mathscr{E} \subset \mathscr{C}_{j}$ and $E=\bigcup \mathscr{E}$, then

$$
\begin{aligned}
\|E\| & \leq \sum_{C \in \mathscr{E}}\|C\| \leq 2 m \sum_{C \in \mathscr{E}_{j}}\left[d\left(C_{j}\right)\right]^{m-1}<2 m \alpha, \\
|E| & \leq \sum_{C \in \mathscr{E}}|C| \leq \frac{\eta_{j}}{\alpha} \sum_{C \in \mathscr{E}_{j}}[d(C)]^{m-1}<\eta_{j} .
\end{aligned}
$$

Now let $\mathscr{C}$ be a disjoint subfamily of $\bigcup_{j} \mathscr{C}_{j}$ such that $\cup \mathscr{C}=\bigcup_{j}\left(\cup \mathscr{C}_{j}\right)$. We define a positive function $\delta_{+}$on $\operatorname{cl} K$ by setting

$$
\delta_{+}(x)= \begin{cases}\delta(x) & \text { if } x \in \operatorname{cl} K-N_{\delta}, \\ \min \{d(C): C \in \mathscr{C}, x \in \operatorname{cl} C\} & \text { if } x \in N_{\delta} .\end{cases}
$$

By classical Cousin's lemma (see [MS, Chapter 4, Theorem 3-1]), there is a $\delta_{+}-$ fine dyadic partition $P=\left\{\left(K_{1}, x_{1}\right), \ldots,\left(K_{p}, x_{p}\right)\right\}$ in $K$ with $\cup P=K$. Let $\mathscr{D}$ consist of all $C \in \mathscr{C}$ such that $C \subset K$ and $K_{i} \subset C$ for some $i=1, \ldots, p$. As $\mathscr{C}$ is disjoint $\mathscr{D}$ is finite, and as $N_{\delta} \subset \operatorname{int}(\bigcup \mathscr{C})$, our definition of $\delta_{+}$ on $N_{\delta}$ implies that $K_{i} \subset \cup \mathscr{D}$ whenever $x_{i} \in N_{\delta}$. If $K_{i}$ meets a $D \in \mathscr{D}$ and $K_{i} \not \subset D$, then $D \subset K_{i}$ because $D$ and $K_{i}$ are dyadic cubes. This is, however, impossible, for by the definition of $\mathscr{D}$, the set $D$ contains a $K_{j}$ disjoint from $K_{i}$. We conclude that for each $i=1, \ldots, p$ either $K_{i} \subset \bigcup \mathscr{D}$ or $K_{i} \cap(\bigcup \mathscr{D})=\varnothing$. Thus after a suitable reordering, $\bigcup \mathscr{D}=K-\bigcup_{i=1}^{q} K_{i}$ for a nonnegative integer $q \leq p$, and $Q=\left\{\left(K_{1}, x_{1}\right), \ldots,\left(K_{q}, x_{q}\right)\right\}$ is a $\delta$-fine dyadic partition in $K$. We complete the proof by showing that $Q$ is $(\tau, \eta)$ approximating for $\tau=1 /(2 m \alpha)$. To this end, let $\mathscr{D}_{1}=\mathscr{D} \cap \mathscr{C}_{1}$ and for $j=1,2, \ldots$, let $\mathscr{D}_{j}=\mathscr{D} \cap \mathscr{C}_{j}-\bigcup_{i=1}^{j-1} \mathscr{D}_{i}$. The family $\mathscr{D}$, being finite, is the union of disjoint families $\mathscr{D}_{1}, \ldots, \mathscr{D}_{k}$. If $D_{j}=\bigcup \mathscr{D}_{j}$ then $\bigcup \mathscr{D}$ is the union of disjoint sets $D_{1}, \ldots, D_{k}$. As $\left\|D_{j}\right\|<1 / \tau$ and $\left|D_{j}\right|<\eta_{j}, j=1, \ldots, k$ our assertion is established.

Lemma 2.4. Let $A$ be a $B V$ set, $x \in \operatorname{int}_{c} A$, and let $\left\{C_{n}\right\}$ be a sequence of dyadic cubes such that $x \in \operatorname{cl} C_{n}, n=1,2, \ldots$, and $\lim d\left(C_{n}\right)=0$. Then $x \in \operatorname{cl}_{e}\left(A \cap C_{n}\right), n=1,2, \ldots$, and

$$
\liminf r\left(A \cap C_{n}\right) \geq \frac{1}{2 m} .
$$


This lemma, which has been proved in [ $\mathrm{P}_{2}$, Lemma 3.6], allows us to apply Lemma 2.3 to $B V$ sets.

Proposition 2.5. Let $\delta$ be a gage in a $B V$ set $A$ and let $\eta$ be a caliber. There is a $\kappa>0$, depending only on the dimension $m$, such that $\Pi(A, \kappa ; \delta, \eta) \neq \varnothing$. Proof. Assume first that $\|A\| \leq 2$. By Lemma 1.1, there is a nondispersed $B V$ set $B \subset A$ with $\|B\| \leq 2$ and $|A-B|<\eta_{1}$. Choose integers $k_{1}, \ldots, k_{m}$ and $h$ so that $K=\prod_{i=1}^{m}\left[k_{i}, k_{i}+2^{h}\right)$ contains $B$. If $x \in \operatorname{cl} K-\operatorname{cl} B$ there is a $\gamma_{x}>0$ with $B \cap U\left(x, \gamma_{x}\right)=\varnothing$. If $x \in \operatorname{int}_{c} B$ and $\beta=1 /(4 m)$, Lemma 2.4 implies the existence of $\gamma_{x}>0$ such that $r(B \cap C)>\beta$ for each dyadic cube $C$ with $x \in \operatorname{cl} C$ and $d(C)<\gamma_{x}$. Since $\operatorname{cl} B-\operatorname{int}_{c} B$ equals to a thin set $\operatorname{cl}_{e} B-\operatorname{int}_{c} B$, setting

$$
\delta^{\circ}(x)= \begin{cases}\gamma_{x} & \text { if } x \in \operatorname{cl} K-\operatorname{cl} B, \\ \min \left\{\gamma_{x}, \delta(x)\right\} & \text { if } x \in \operatorname{int}_{c} B, \\ 0 & \text { if } x \in \operatorname{cl} B-\operatorname{int}_{c} B,\end{cases}
$$

defines a gage $\delta^{\circ}$ in $K$. Let $\eta^{\circ}=\left\{\eta_{2}, \eta_{3}, \ldots\right\}$ and use Lemma 2.3 to find a $\delta^{\circ}$-fine $\left(\tau, \eta^{\circ}\right)$-approximating dyadic partition $Q$ in $K$. Then $P=$ $\left\{(B \cap L, x):(L, x) \in Q, x \in\right.$ int $\left._{c} B\right\}$ is a $\delta$-fine $\beta$-regular partition in B and $B-\bigcup P=B \cap(K-\bigcup Q)$. As $K-\bigcup Q$ is the union of disjoint $B V$ sets $D_{2}, \ldots, D_{k}$ with $\left\|D_{j}\right\|<1 / \tau$ and $\left|D_{j}\right|<\eta_{j}, j=2, \ldots, k$, an easy calculation shows that $P \in \Pi(A, \kappa ; \delta, \eta)$ for $\kappa=\min \{\beta, \tau /(1+2 \tau)\}$.

An arbitrary $B V$ set $A$ is the union of disjoint $B V$ sets $A_{1}, \ldots, A_{n}$ whose perimeters are less than or equal to 2 . For $i=0, \ldots, n-1$, let $\eta^{i}=\left\{\eta_{n j-i}\right\}_{j}$ and use the first part of the proof to find a $P_{i} \in \Pi\left(A_{i}, \kappa ; \delta, \eta^{i}\right)$. It is clear that $P=\bigcup_{i=1}^{n} P_{i}$ belongs to $\Pi(A, \kappa ; \delta, \eta)$.

\section{THE INTEGRAL}

If $A$ is a $B V$ set and $f$ is a function on $\operatorname{cl}_{e} A$, we set

$$
\sigma(f, P)=\sum_{i=1}^{p} f\left(x_{i}\right)\left|A_{i}\right|
$$

for each partition $P=\left\{\left(A_{1}, x_{1}\right), \ldots,\left(A_{p}, x_{p}\right)\right\}$ in $A$.

Definition 3.1. Let $A$ be a $B V$ set and let $f$ be a function on $\operatorname{cl}_{e} A$. We say that $f$ is integrable in $A$ if there is a real number $I$ having the following property: given $\varepsilon>0$, we can find a gage $\delta$ in $A$ and a caliber $\eta$ so that $|\sigma(f, P)-I|<\varepsilon$ for each $P \in \Pi(A, \varepsilon ; \delta, \eta)$.

The family of all integrable functions in a $B V$ set $A$ is denoted by $\mathscr{I}(A)$. It follows from Proposition 2.5 that the number $I$ of Definition 3.1 is determined uniquely by $f \in \mathscr{I}(A)$. We call it the integral of $f$ over $A$, denoted by $\int_{A} f$.

Let $A$ be a $B V$ set. A division of $A$ is a finite disjoint family of $B V$ sets whose union is $A$. A function $F$ on $B V_{A}$ is called:

(i) additive if $F(A)=\sum_{D \in \mathscr{D}} F(D)$ for each division $\mathscr{D}$ of $A$;

(ii) continuous if given $\varepsilon>0$, there is a $\nu>0$ such that $|F(B)|<\varepsilon$ for each $B \in B V_{A}$ with $\|B\|<1 / \varepsilon$ and $|B|<\nu$. 
Proposition 3.2. Let $A \in B V$ and $f \in \mathcal{I}(A)$. Then $f_{B}=f\left\lceil\mathrm{cl}_{e} B\right.$ belongs to $\mathscr{I}(B)$ for each $B \in B V_{A}$, and the map $F: B \mapsto \int_{B} f_{B}$ is an additive continuous function on $B V_{A}$.

Proof. Choose a positive $\varepsilon \leq \kappa$ where $\kappa$ is the constant from Proposition 2.5. There is a gage $\delta$ in $A$ and a caliber $\eta$ such that $\left|\sigma(f, P)-\int_{A} f\right|<\varepsilon / 2$ for each $P \in \Pi(A, \varepsilon ; \delta, \eta)$.

Let $\eta^{e}=\left\{\eta_{2 j}\right\}$ and $\eta^{o}=\left\{\eta_{2 j-1}\right\}$. Given a $B \in B V_{A}$, select partitions $Q_{1}$ and $Q_{2}$ in $\Pi\left(B, \varepsilon ; \delta, \eta^{e}\right)$. Using Proposition 2.5, find a partition $Q \in$ $\Pi\left(A-B, \varepsilon ; \delta, \eta^{o}\right)$. Now $P_{i}=Q_{i} \cup Q, i=1,2$, belongs to $\Pi(A, \varepsilon ; \delta, \eta)$ and

$$
\begin{aligned}
\left|\sigma\left(f, Q_{1}\right)-\sigma\left(f, Q_{2}\right)\right| & =\left|\sigma\left(f, P_{1}\right)-\sigma\left(f, P_{2}\right)\right| \\
& \leq\left|\sigma\left(f, P_{1}\right)-\int_{A} f\right|+\left|\int_{A} f-\sigma\left(f, P_{2}\right)\right|<\varepsilon .
\end{aligned}
$$

Thus $f_{B}$ belongs to $\mathscr{I}(B)$ since it satisfies Cauchy's test for integrability (cf. $\left[\mathrm{P}_{2}\right.$, Lemma 7.4]).

If $\left\{A_{1}, \ldots, A_{n}\right\}$ is a division of $A$, let $\eta^{k}=\left\{\eta_{n j-k}\right\}_{j}, k=0, \ldots, n-1$. By the first part of the proof and Proposition 2.5, there is a $P_{k} \in \Pi\left(A_{k}, \varepsilon ; \delta, \eta^{k}\right)$ such that $\left|\sigma\left(f, P_{k}\right)-F\left(A_{k}\right)\right|<\varepsilon /(2 n)$. Because $P=\bigcup_{k=1}^{n} P_{k}$ belongs to $\Pi(A, \varepsilon ; \delta, \eta)$, we have

$$
\left|F(A)-\sum_{k=1}^{n} F\left(A_{k}\right)\right| \leq|F(A)-\sigma(f, P)|+\sum_{k=1}^{n}\left|\sigma\left(f, P_{k}\right)-F\left(A_{k}\right)\right|<\varepsilon,
$$

and the additivity of $F$ follows from the arbitrariness of $\varepsilon$.

Choose a $B \in B V_{A}$ with $\|B\|<1 / \varepsilon$ and $|B|<\eta_{1}$. Let $C=A-B, \eta^{\circ}=$ $\left\{\eta_{2}, \eta_{3}, \ldots\right\}$, and let $Q \in \Pi\left(C, \varepsilon ; \delta, \eta^{\circ}\right)$ be such that $|\sigma(f, Q)-F(Q)|<$ $\varepsilon / 2$. Since $Q$ also belongs to $\Pi(A, \varepsilon ; \delta, \eta)$, we obtain

$$
|F(B)|=|F(A)-F(C)| \leq|F(A)-\sigma(f, Q)|+|\sigma(f, Q)-F(C)|<\varepsilon
$$

which establishes the continuity of $F$.

Theorem 3.3. Let $A \in B V$ and let $f$ be a function on $\operatorname{cl}_{e} A$. Then $f \in \mathscr{I}(A)$ if and only if there is an additive continuous function $F$ on $B V_{A}$ which satisfies the following condition: given $\varepsilon>0$, we can find a gage $\delta$ in $A$ so that

$$
\sum_{i=1}^{p}\left|f\left(x_{i}\right)\right| A_{i}\left|-F\left(A_{i}\right)\right|<\varepsilon
$$

for each $\varepsilon$-regular $\delta$-fine partition $\left\{\left(A_{1}, x_{1}\right), \ldots,\left(A_{p}, x_{p}\right)\right\}$ in $A$. In particular, we have $\int_{A} f=F(A)$.

Proof. Assume first that $f \in \mathscr{I}(A)$ and let $F(B)=\int_{B} f$ for each $B \in B V_{A}$. By Proposition 3.2, $F$ is an additive continuous function on $B V_{A}$. Choose a positive $\varepsilon \leq \kappa$ where $\kappa$ is the constant from Proposition 2.5. There is a gage $\delta$ in $A$ and a caliber $\eta$ such that $|\sigma(f, P)-F(A)|<\varepsilon / 3$ for each $P \in$ $\Pi(A, \varepsilon ; \delta, \eta)$. Let $\eta^{e}=\left\{\eta_{2 j}\right\}$ and $\eta^{o}=\left\{\eta_{2 j-1}\right\}$. It follows from Proposition 2.5 that each $\varepsilon$-regular $\delta$-fine partition in $A$ is a subset of a partition from $\Pi\left(A, \varepsilon ; \delta, \eta^{o}\right)$. Thus it suffices to prove the required inequality for a partition $\left\{\left(A_{1}, x_{1}\right), \ldots,\left(A_{p}, x_{p}\right)\right\}$ in $\Pi\left(A, \varepsilon ; \delta, \eta^{o}\right)$. 
To this purpose let $\eta^{i}=\left\{\eta_{2 p j-2 i}\right\}_{j}, i=0, \ldots, p-1$, and use Propositions 2.5 and 3.2 to find a $P_{i} \in \Pi\left(A_{i}, \varepsilon ; \delta, \eta^{i}\right)$ so that $\left|\sigma\left(f, P_{i}\right)-F\left(A_{i}\right)\right|<\varepsilon /(3 p)$. After a suitable reordering there is a nonnegative integer $k \leq p$ such that $f\left(x_{i}\right)\left|A_{i}\right| \geq F\left(A_{i}\right)$ for $i=1, \ldots, k$ and $f\left(x_{i}\right)\left|A_{i}\right|<F\left(A_{i}\right)$ for $i=k+$ $1, \ldots, p$. By our choice of the $\eta^{i}$ 's, it is easy to see that

$$
\begin{aligned}
& P_{+}=\left\{\left(A_{1}, x_{1}\right), \ldots,\left(A_{k}, x_{k}\right)\right\} \cup \bigcup_{i=k+1}^{p} P_{i}, \\
& P_{-}=\left\{\left(A_{k+1}, x_{k+1}\right), \ldots,\left(A_{p}, x_{p}\right)\right\} \cup \bigcup_{i=1}^{k} P_{i}
\end{aligned}
$$

belong to $\Pi(A, \varepsilon ; \delta, \eta)$. Hence

$$
\begin{aligned}
\frac{\varepsilon}{3} & >\sigma\left(f, P_{+}\right)-F(A)=\sum_{i=1}^{k}\left|f\left(x_{i}\right)\right| A_{i}\left|-F\left(A_{i}\right)\right|+\sum_{i=k+1}^{p}\left[\sigma\left(f, P_{i}\right)-F\left(A_{i}\right)\right] \\
& \geq \sum_{i=1}^{k}\left|f\left(x_{i}\right)\right| A_{i}\left|-F\left(A_{i}\right)\right|-\frac{\varepsilon(p-k)}{3 p}
\end{aligned}
$$

and similarly

$$
\frac{\varepsilon}{3}>\sum_{i=k+1}^{p}\left|f\left(x_{i}\right)\right| A_{i}\left|-F\left(A_{i}\right)\right|-\frac{\varepsilon k}{3 p} .
$$

The desired inequality is obtained by adding the last two inequalities.

Conversely, suppose that an additive continuous function $F$ on $B V_{A}$ satisfying the conditions of the theorem exists. Choose an $\varepsilon>0$ and find a gage $\delta$ in $A$ so that

$$
\sum_{i=1}^{p}\left|f\left(x_{i}\right)\right| A_{i}\left|-F\left(A_{i}\right)\right|<\frac{\varepsilon}{2}
$$

for each $\varepsilon$-regular $\delta$-fine partition $\left\{\left(A_{1}, x_{1}\right), \ldots,\left(A_{p}, x_{p}\right)\right\}$ in $A$. Since $F$ is continuous, there is a caliber $\eta$ such that $|F(B)|<\varepsilon 2^{-j-1}$ for each $B \in$ $B V_{A}$ with $\|B\|<1 / \varepsilon$ and $|B|<\eta_{j}, j=1,2, \ldots$. Now given a partition $P=\left\{\left(A_{1}, x_{1}\right), \ldots,\left(A_{p}, x_{p}\right)\right\}$ in $\Pi(A, \varepsilon ; \delta, \eta)$, the set $A-\bigcup P$ is the union of disjoint $B V$ sets $B_{1}, \ldots, B_{k}$ such that $\left\|B_{j}\right\|<1 / \varepsilon$ and $\left|B_{j}\right|<\eta_{j}$ for $j=1, \ldots, k$. Thus

$$
|\sigma(f, A)-F(A)| \leq \sum_{i=1}^{p}\left|f\left(x_{i}\right)\right| A_{i}\left|-F\left(A_{i}\right)\right|+\sum_{j=1}^{k}\left|F\left(B_{j}\right)\right|<\frac{\varepsilon}{2}+\sum_{j=1}^{k} \varepsilon 2^{-j-1}<\varepsilon,
$$

and we see that $f \in \mathscr{I}(A)$ and $\int_{A} f=F(A)$.

From the previous theorem and $\left[\mathrm{P}_{2}\right.$, Propositions 7.7 and 7.8$]$ we obtain immediately the following corollary.

Corollary 3.4. The integral of Definition 3.1 coincides with the tight variational integral defined in $\left[\mathrm{P}_{2}\right.$, Remark 5.2, 4(a)].

Each equivalence class of $B V$ sets modulo, the sets of Lebesgue measure zero contains a unique essentially closed set. In dimension one such a set is 
a finite union of nondegenerate compact intervals (see $[\mathrm{V}, \S 6])$. This observation enables us to present a particularly simple definition of the integral when $m=1$-an assumption we shall make for the remainder of the paper.

By an interval we mean a compact interval $[a, b] \subset \mathbf{R}$ with $a<b$. A figure is a finite union of intervals, and the family of all figures is denoted by $\mathscr{F}$. We say that figures $A$ and $B$ overlap if $|A \cap B|>0$. An $\mathscr{F}$-partition of a figure $A$ is a collection $\left\{\left(A_{1}, x_{1}\right), \ldots,\left(A_{p}, x_{p}\right)\right\}$ such that $A_{1}, \ldots, A_{p}$ are nonoverlapping figures, $\bigcup_{i=1}^{p} A_{i}=A$, and $x_{i} \in A_{i}, i=1, \ldots, p$. The $\varepsilon$ regular and $\delta$-fine $\mathscr{F}$-partitions of a figure $A$ are defined in the obvious way. For $\mathscr{F}$-partitions, the obvious meaning is also given to the symbol $\sigma(f, P)$.

Proposition 3.5. Let $f$ be a function defined on a figure $A$. Then $f \in \mathscr{I}(A)$ with $\int_{A} f=I$ if and only if for each $\varepsilon>0$ there is a positive gage $\delta$ in $A$ such that $|\sigma(f, P)-I|<\varepsilon$ for every $\varepsilon$ - regular $\delta$-fine $\mathscr{F}$-partition $P$ of $A$.

Proof. Let $f \in \mathscr{J}(A)$ and $\varepsilon>0$. There is a gage $\delta$ in $A$ and a caliber $\eta$ such that $\left|\sigma(f, Q)-\int_{A} f\right|<\varepsilon / 2$ for each $Q \in \Pi(A, \varepsilon / 2 ; \delta, \eta)$. As $m=1$, the gage $\delta$ is positive outside a countable set $N=\left\{z_{2}, z_{3}, \ldots\right\}$. With no loss of generality we may assume that $\eta_{j}\left|f\left(z_{j}\right)\right| \leq \varepsilon 2^{-j}$ for $j=2,3, \ldots$. We define a positive gage $\delta^{\circ}$ in $A$ by setting

$$
\delta^{\circ}= \begin{cases}\delta(x) & \text { if } x \in A-N \\ \eta_{j} & \text { if } x=z_{j}, j=2,3, \ldots,\end{cases}
$$

and choose an $\varepsilon$-regular $\delta^{\circ}$-fine $\mathscr{F}$-partition $P=\left\{\left(A_{1}, x_{1}\right), \ldots,\left(A_{p}, x_{p}\right)\right\}$ of $A$. Clearly, $Q=\left\{\left(\right.\right.$ int $\left.\left.A_{i}, x_{i}\right): x_{i} \in A-N\right\}$ is an $\varepsilon$-regular $\delta$-fine partition in $A$. To show that $Q$ is also $(\varepsilon / 2, \eta)$-approximating, let $B_{1}=\bigcup_{i=1}^{p}\left(\mathrm{bd} A_{i}\right)$ and $B_{j}=\bigcup\left\{\right.$ int $\left.A_{i}: x_{i}=z_{j}\right\}, j=2,3, \ldots$ The sets $B_{j}$ are disjoint, $\left|B_{j}\right|<\eta_{j}$ for $j=1,2, \ldots$, and $A-\cup Q=\bigcup_{j=1}^{k} B_{j}$ for an integer $k \geq 1$. Moreover, $\left\|B_{j}\right\|<2 / \varepsilon$ since $r\left(A_{i}\right)>\varepsilon$ and the map $i \mapsto x_{i}$ is at most two-to-one. Thus

$$
\left|\sigma(f, P)-\int_{A} f\right| \leq \sum_{j=2}^{k}\left|f\left(z_{j}\right)\right| \cdot\left|B_{j}\right|+\left|\sigma(f, Q)-\int_{A} f\right|<\sum_{j=2}^{k} \varepsilon 2^{-j}+\frac{\varepsilon}{2}<\varepsilon .
$$

To prove the converse, for every figure $B \subset A$, denote by $F(B)$ a real number which satisfies the following condition: given an $\varepsilon>0$, there is a positive gage $\delta$ in $B$ such that $|\sigma(f, Q)-F(B)|<\varepsilon$ for each $\varepsilon$-regular $\delta$-fine $\mathscr{F}$-partition $Q$ of $B$. If the condition of the proposition is satisfied, it is completely routine to show that a unique number $F(B)$ exists for each figure $B \subset A$, and that the map $B \mapsto F(B)$ extends uniquely to an additive function $F$ on $B V_{A}$. The following Henstock lemma (cf. $\left[\mathrm{P}_{1}\right.$, Lemma 2.5$\left.]\right)$ is also obtained by a standard argument.

Given $\varepsilon>0$, there is a positive gage $\delta$ in $A$ such that

$$
\sum_{i=1}^{p}\left|f\left(x_{i}\right)\right| A_{i}\left|-F\left(A_{i}\right)\right|<\varepsilon
$$

for each $\varepsilon$-regular $\delta$-fine partition $\left\{\left(A_{1}, x_{1}\right), \ldots,\left(A_{p}, x_{p}\right)\right\}$ in $A$.

Therefore, in view of Theorem 3.3 , it suffices to show that $F$ is a continuous function. Choose an interval $[a, b]$ containing $A$, and for each $x \in A$ let $\varphi(x)=F(A \cap[a, x])$. If $B \in B V_{A}$ and $\left[a_{i}, b_{i}\right], i=1, \ldots, n$, are the 
connected components of $\operatorname{cl}_{e} B$, then $F(B)=\sum_{i=1}^{n}\left[\varphi\left(b_{i}\right)-\varphi\left(a_{i}\right)\right]$. Now given $\varepsilon>0$ and $x \in A$, it follows from the Henstock lemma quoted above that there is a positive $\nu<\varepsilon /[|f(x)|+1]$ such that $|f(x)(y-x)-[\varphi(y)-\varphi(x)]|<\varepsilon$ for each $y \in A$ with $|y-x|<\nu$. Hence

$$
|\varphi(y)-\varphi(x)|<|f(x)| \cdot|y-x|+\varepsilon<2 \varepsilon
$$

whenever $y \in A$ and $|y-x|<\nu$, and we see that $\varphi$ is continuous at $x$. It follows that $\varphi$ is uniformly continuous in $A$, and this implies the continuity of $F$.

Note. In dimension one, the integral lies properly in between the Lebesgue and Denjoy-Perron integrals (see $\left[\mathrm{P}_{2}\right.$, Proposition 6.8]). A detailed analysis of this situation is given in the forthcoming paper [B-G-P].

\section{REFERENCES}

[B-G-P] B. Bongiorno, M. Giertz, and W. F. Pfeffer, Some nonabsolutely convergent integrals in the real line (to appear).

[C-T] C. Congedo and I. Tamanini, Note sulla regolarità dei minimi di funzionali del tipo dell'area, Rend. Accad. Naz. Sci. XL Mem. Mat. (5) 106 (1988), 239-257.

[Fa] K.J. Falconer, The geometry of fractal sets, Cambridge Univ. Press, Cambridge, 1985.

[Fe] H. Federer, Geometric measure theory, Springer-Verlag, New York, 1969.

$[\mathrm{HH}]$ E. J. Howard, Analyticity of almost everywhere differentiable functions, Proc. Amer. Math. Soc. 110 (3), 745-753.

[M-M] U. Massari and M. Miranda, Minimal surfaces in codimension one, North-Holland, Amsterdam, 1984.

[MS] E. J. McShane, Unified integration, Academic Press, New York, 1983.

$\left[\mathrm{P}_{1}\right] \quad$ W. F. Pfeffer, A Riemann type integration and the fundamental theorem of calculus, Rend. Circ. Mat. Palermo (2) 36 (1987), 482-506.

$\left[\mathrm{P}_{2}\right] \quad$ W. F. Pfeffer, The Gauss-Green theorem, Adv. in Math. (to appear).

[S] S. Saks, Theory of the integral, Dover, New York, 1964.

[T-G] I. Tamanini and C. Giacomelli, Approximation of Caccioppoli sets, with applications to problems in image segmentation, Ann. Univ. Ferrara Sez. VII (N.S.) 35 (1989), 187-213.

[V] A. I. Volpert, The spaces BV and quasilinear equations, Math. USSR-Sb. 2 (1967), 225-267.

Department of Mathematics, University of California, Davis, California 95616 